\title{
Responses to Economic Games of Cooperation and Conflict in Squirrel Monkeys (Saimiri boliviensis)
}

\author{
Gillian L. Vale ${ }^{\mathrm{a}, \mathrm{b}}$, Lawrence E. Williams ${ }^{\mathrm{a}}$, Steven J. Schapiro ${ }^{\mathrm{a,c}}$, \\ Susan P. Lambeth ${ }^{\mathrm{a}}$ and Sarah F. Brosnan ${ }^{\mathrm{a}, \mathrm{b}}$
}

\author{
${ }^{a}$ Michale E. Keeling Center for Comparative Medicine and Research, The University of Texas MD Anderson Cancer Center, \\ Bastrop, TX, USA \\ ${ }^{b}$ Department of Psychology, Language Research Center, Neuroscience Institute and Center for Behavioral Neuroscience, \\ Georgia State University, Atlanta, GA, USA \\ ${ }^{\mathrm{c}}$ Department of Experimental Medicine, University of Copenhagen, Copenhagen, Denmark \\ *Corresponding author (Email: gillvale3@ hotmail.com)
}

Citation - Vale, G. L., Williams, L. E., Schapiro, S. J., Lambeth, S. P., \& Brosnan, S. F. (2019). Responses to economic games of cooperation and conflict in squirrel monkeys (Saimiri boliviensis). Animal Behavior and Cognition, 6(1), 32-47. https://doi.org/10.26451/abc.06.01.03.2019

\begin{abstract}
Games from experimental economics have provided insights into the evolutionary roots of social decision making in primates and other species. Multiple primate species' abilities to cooperate, coordinate and anti-coordinate have been tested utilizing variants of these simple games. Past research, however, has focused on species known to cooperate and coordinate in the wild. To begin to address the degree to which cooperation and coordination may be a general ability that manifests in specific contexts, the present study assessed the decisions of squirrel monkeys (Saimiri boliviensis; $N=10$ ), a species not known for their cooperative behavior in these games. Pairs of monkeys were presented with the Assurance Game (a coordination game), the Hawk-Dove Game (an anti-coordination game) and the Prisoner's Dilemma (a cooperation game with a temptation to defect). We then compared squirrel monkeys' performance to existing data on capuchin monkeys (Sapajus [Cebus] apella), a closely related species that routinely cooperates, to determine what, if any, differences in decision making emerged. Some pairs of both species found the payoff-dominant Nash Equilibrium (NE) in the coordination game, but failed to find the NE in subsequent games. Our results suggest that, like capuchins, squirrel monkeys coordinate their behavior with others, suggesting that such mutual outcomes occur in at least some contexts, even in species that do not routinely cooperate.
\end{abstract}

Keywords - Assurance Game, Cooperation, Coordination, Hawk-Dove Game, Prisoner's Dilemma

Experimental evidence derived from captive studies shows that several ape and monkey species not only cooperate, but also appear to understand at least some of the contingencies of cooperation. Ape studies indicate that chimpanzees (Pan troglodytes), bonobos (Pan paniscus) and orangutans (Pongo pygmaeus) will work with a conspecific for mutual rewards (Pongo pygmaeus: Chalmeau, Lardeux, Brandibas, \& Gallo, 1997; Pan troglodytes: Crawford, 1937; 1941; Hare, Melis, Woods, Hastings, \& Wrangham, 2007; Melis, Hare, \& Tomasello, 2006a; Melis, Hare, \& Tomasello, 2006b; Pan paniscus: Hare et al., 2007). Whether this cooperation occurs appears to be moderated by factors such as tolerance levels between partners, reward distributions, and the individuals involved. Chimpanzees, for example, preferentially recruit partners with a previous track record of successful collaboration (Melis et al., 2006a). They are also less likely to cooperate when resources can be monopolized by one individual or are asymmetric (Greenberg, Hamann, Warneken, \& Tomasello, 2010; Hare et al., 2007). Similar tasks indicate 
that capuchins (Sapajus [Cebus] apella: de Waal \& Davis, 2003; reviewed in Brosnan, 2011) and cottontop tamarins (Saguinus oedipus: Cronin, Kurian, \& Snowdon, 2005) also understand at least some of the contingencies of cooperation.

Previous research on decision making in primates has focused on species that cooperate and coordinate in the wild. However, just because species are not known for their cooperative tendencies does not mean that they cannot cooperate under the right conditions. It is important to study a variety of species, including those that have not been observed cooperating in the wild, to determine when cooperation is specific to certain species and when it is triggered by context, irrespective of species' typical behaviors. One way to do this is to expose species that differ in the extent to which they cooperate to the same experimental conditions to see what they do. We did so for the present study, using economic game paradigms to test squirrel monkeys (Saimiri boliviensis), a species not known to cooperate extensively, in a variety of decision-making contexts. Squirrel monkeys were tested on the Assurance game, a coordination game for which capuchin monkeys, chimpanzees, and rhesus monkeys find the highest-paying coordinated outcome (Brosnan et al., 2011; 2018; Brosnan, Wilson, \& Beran, 2012; Smith, Leverett, Wilson, \& Brosnan, unpublished data) and two other games testing anti-coordination and cooperation with a temptation to defect (see details below), both games for which capuchins tend to play the Nash Equilibrium. We directly compared the squirrel monkeys' results to existing data on capuchin monkeys, a closely related, sympatric species known to cooperate both in the wild and in captivity, to determine what, if any, differences in decision making emerged between the two species.

Capuchins (both Sapajus and Cebus; Alfaro, Silva, \& Rylands, 2012) and squirrel monkeys make an excellent comparison as they are con-familial (sharing the phylogenetic family Cebidae) and sympatric, found over the same parts of South and Central America in the same ecological conditions (Boinski, 1999; Boinski et al., 2002). In addition, they are similar in morphology (Fleagle, 1999), although capuchins are larger in size (Marroig, 2007). Both also have relatively large brains for their body size (although capuchins' are slightly larger; Hartwig, Rosenberger, Norconk, \& Owl, 2011), a measure that has been argued to be associated with cognitive performance (Reader \& Laland, 2002; although see Deaner, Isler, Burkart, \& van Schaik, 2007, for an argument that absolute brain size is more important). Squirrel monkeys live in larger groups than capuchins (squirrel monkeys: 25-75 monkeys; Boinski, 1999; capuchin monkeys: 12-27 monkeys; Fragaszy, Visalberghi, \& Fedigan, 2004), but, if anything, this might indicate selection for more complex social lives in squirrel monkeys.

One key difference is in their level of cooperation. Capuchins cooperate in a variety of contexts. In the wild, they show group territorial behavior (e.g., Scarry, 2017), reciprocate grooming (di Bitetti, 1997; Manson, Navarrete, Silk, \& Perry, 2004), transfer food (infrequently; Rose, 1997), and show some level of group hunting (although it is not terribly coordinated; Rose, 1997), as well as group predator defense (Perry, Manson, Dower, \& Wikbert, 2003). In captivity, there is evidence that capuchins recognize the need for a partner in collaborative tasks, adjusting their behavior based on the presence or absence of a partner, depending on whether they can see him or her, and the quality and distribution of rewards (reviewed in Brosnan, 2011). In addition, food transfer correlates positively with previous transfer rates, and is influenced by cooperative effort and the monkeys' relationship quality (de Waal, 2000; de Waal \& Berger, 2000; Sabbatini, Vizioli, Visalberghi, \& Schino, 2012).

Even though there has been more research effort with capuchins than with squirrel monkeys, it is still notable that we find very few references to squirrel monkey cooperation in the field. Supporting the possibility of cooperative behavior, S. boliviensis form coalitions and alliances in the context of migration and resource defense (Boinski et al., 2002) and are known to transfer food in captivity, despite the lack of evidence of food sharing in the wild (Stevens, 2004). In addition, squirrel monkeys reconcile, a behavior that seems to indicate the importance of maintaining one's social connections (Pereira, Schill, \& Charles, 2000; capuchins also reconcile in some contexts; Verbeek \& de Waal, 1997). However, unlike capuchins, squirrel monkeys show no evidence of responding to inequitable outcomes (Freeman et al., 2013; Talbot, Freeman, Williams, \& Brosnan, 2011), a behavior that has been linked to cooperation (Brosnan \& de Waal, 2014). 
One of the challenges of comparative work is that paradigms must be directly comparable across species or we cannot draw conclusions about the differences, or lack thereof, between those species. For this study, we used games derived from experimental economics, an approach that simplifies decisionmaking situations to two discrete choices, with payoffs based on both subjects' decisions. Importantly for this work, these games produced results that were comparable across species, and allowed different decision scenarios to be created by altering only the payoffs, keeping the rest of the procedure constant. This allowed us to test decisions across different contexts using the same format (c.f., Smith, Watzek, \& Brosnan, 2018). Indeed, species ranging from rats to pigeons to primates have been tested with these tasks, revealing that species sometimes find cooperative outcomes, whether or not they are known to routinely cooperate in other contexts (e.g., Macaca mulatta: Brosnan et al., 2012; Cyanocitta cristata: Stevens \& Stephens, 2004; Rattus norvegicus: Li \& Wood, 2017), and sometimes they find uncooperative outcomes (e.g., Rattus norvegicus: Gardner, Corbin, Beltramo, \& Nickell, 1984; Columba livia: Green, Price, \& Hamburger, 1995).

We tested squirrel monkeys on three economic games, the Assurance, or Stag-Hunt, game (AG; a coordination game in which it pays to perform the same action as a conspecific), the Hawk-Dove game (HDG; an anti-coordination game in which incentives are not aligned, at least in the short term) and the Prisoners-Dilemma game (PDG; a cooperation game that heightens the incentives to defect from a mutually beneficial outcome). The first of these three games, the AG, provides a simple model of low-cost human coordination (Skyrms, 2003). In this game, paired participants choose between two options, Stag and Hare. If both participants choose Stag, they receive the highest payoff, whereas any partner that plays Hare receives a lower payoff irrespective of what their partner plays (playing Hare is risk dominant, because the subject receives a reward regardless of what their partner plays; see Figure 1). Thus, there are two coordinated Nash Equilibria (NE; the NE is the outcome for which neither player can do better by playing a different option given their partner's choice), coordinating on Stag (the payoff dominant NE) or coordinating on Hare. Capuchin monkeys, chimpanzees, rhesus monkeys and humans all display some capacity to find the payoff dominant NE in at least some contexts, and even in these very simple games, we can begin to tease apart different cognitive mechanisms that can allow species to reach the same outcomes. Rhesus monkeys, for example, appeared to converge on Stag-Stag through individual preferences for the highest paying option (Parrish, Brosnan, Wilson, \& Beran, 2014), whereas capuchins apparently employed a matching strategy that required that they be able to see their partner's choices to converge on Stag-Stag (Brosnan et al., 2012).
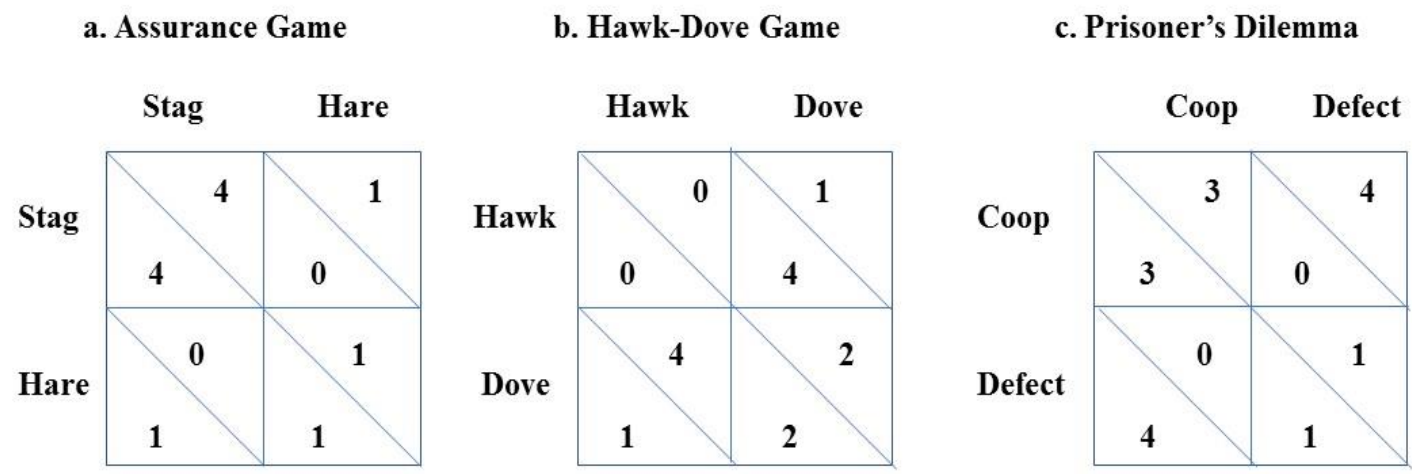

Figure 1. Payoff matrices for the a) Assurance Game, b) Hawk-Dove Game, and c) Prisoner's Dilemma Game.

The flip side of coordination is anti-coordination, which is modelled in the second game, the HDG. In this game, individuals can play Dove, yielding to their partner, or Hawk, fighting for the resource. One participant benefits when they play Hawk and the other plays Dove; however, if both play Hawk, the resources are lost (see Figure 1). There are two asymmetric Nash equilibria in this game (i.e., one player plays Hawk and the other Dove), and the payoff dominant strategy is to alternate the NE (i.e., take turns 
playing Hawk and Dove). In previous work, both capuchin and rhesus monkeys found the NE, but only in a sequential game when they could see what their partner was playing, and only humans found the alternating NE (Brosnan et al., 2018).

Although in situations like the AG coordination may be easy to establish, as there is no incentive to defect, in real life there is often tension between defecting and working with others. In the PDG, individuals do best in the long run by cooperating, but, in the short term, they do best by defecting, no matter what their partner plays (and indeed, mutual defection is the NE; see Figure 1). Most studies have found that animals primarily defect in the PDG (Clements \& Stephens, 1995; Green et al., 1995). However, Haroush and Williams (2015) found that, whereas mutual defection was the most common choice, rhesus monkeys were more likely to cooperate on trials immediately following those in which their partner had cooperated, indicating some understanding of the benefits of cooperation. Our recent work on capuchin monkeys found both mutual cooperation and mutual defection, showing that despite the pull to maximize short-term rewards, some capuchins, in some contexts, maximize long-term outcomes (Smith et al., unpublished data).

The goal of our study was to compare squirrel monkeys' decisions in these three games to our earlier results from capuchins (as well as from rhesus monkeys, chimpanzees, and humans). One important caveat is in order; in the previous games in capuchins, we included both a manual version of the tasks, in which subjects made a choice by choosing one of two tokens and returning it to an experimenter, and a computerized version of the tasks, in which subjects made a choice by choosing one of two icons on a computer screen using a joystick. A major difference emerged in that capuchins were much more likely to develop a consistent pattern of play in the computerized task (although when they developed a pattern in the manual task, it was always the same strategy as they used in the computerized task). We hypothesized that this was due to a combination of factors that improve learning (Wolfe, 1934), including the ability to include more trials per session, with a shorter inter-trial-interval, and a much shorter delay between choice and reward in computerized tests (because computers are faster than human experimenters; see Smith et al., 2018). In addition, there were no experimenters present, and this absence may have reduced extraneous information that biased or misled subjects. Unfortunately, our squirrel monkeys were not trained to use computerized testing and so we could only present them with the manual version of these tasks. Therefore, when comparing results below, we compare the capuchins' outcomes on both manual and computerized versions, and specify which results relate to which paradigm. However, if squirrel monkeys are similar to capuchins and perform better on the computerized tests, using the manual tests weakens our ability to detect cooperation or NE play in these games. Nonetheless, because capuchins can find the outcomes in manual tasks as well, we felt it was important to assess, to the best of our ability, whether squirrel monkeys show any ability to find a stable pattern of play in these games and to draw the comparison with capuchins' results.

We predicted that the squirrel monkeys would not be able to find coordinated outcomes in the AG. Despite their food sharing in captive situations, and their forming coalitions and alliances, reconciling, and learning socially from one another (Boinski et al., 2002; Hopper, Holmes, Williams, \& Brosnan, 2013; Stevens, 2004), they do not show extensive cooperation in the wild, or many of the behaviors related to cooperation (i.e., responses to inequity: Talbot et al., 2011). Considering the other games, if the squirrel monkeys did find the coordinated outcome in the AG, then in principle, they should be able to do so in the other games as well, for instance, through associative learning. However, we predicted that they would have more difficulty finding outcomes that require anti-coordination or costly cooperation because they are less obvious (i.e., the difference in number of rewards between the NE and non-NE outcomes is smaller), require inhibition of the tendency to choose the sometimes higher-paying option, and do not have a joint bestpaying outcome. Coordinating in the AG, but failing to find the NE in subsequent games, would be tentative evidence that they were better at learning to achieve coordinated outcomes than finding consistent patterns of play in games of conflict (HDG) and games of cooperation that include elements of both conflict and cooperation (PDG). 


\section{Methods}

\section{Ethics Statement}

This research was conducted at the Michale E. Keeling Center for Comparative Medicine and Research, The University of Texas MD Anderson Cancer Center, Bastrop, Texas (KCCMR). The KCCMR is fully accredited by AAALAC International. All animal experiments were conducted according to the provisions of the Animal Welfare Act, PHS Animal Welfare Policy, and the principles of the NIH Guide for the Care and Use of Laboratory Animals. All procedures were approved by the Institutional Animal Care and Use Committee of MD Anderson (IACUC\# 149-RN01). Squirrel monkeys had access to their living enclosures throughout testing and had ad lib access to water and food, including during test sessions. Subjects were not isolated during testing or for training.

\section{Subjects}

Subjects consisted of three female pairs and two male pairs of Bolivian squirrel monkeys $(N=10$ subjects, see Table 1) housed at the KCCMR. All pairs were tested on all three games, except for one male pair (PE-RS) that did not participate in the PDG due to injury. Squirrel monkeys were never water or food deprived for this study, and the test rewards were in addition to their regular daily diet. Subjects participated on a voluntary basis by choosing to enter the test enclosure attached to their home enclosure and take tokens from the experimenter. As they retained access to their home enclosures, subjects could end testing at any point by walking away. Pairs were tested on consecutive working days, up to twice daily, and monkeys could choose not to participate on any given day/session. Veterinary procedures also took precedence over testing. Where testing was interrupted, it was resumed the following day. The three female pairs had previous experience in an inequity aversion exchange task; the male pairs were naïve to behavioral experiments. The four males were already pair housed, and the six females from two larger social groups were temporarily pair housed with members of their social groups during this investigation. Females with close social ties were paired together to minimize stress and potential conflict. Testing was limited to these pairings, rather than testing each individual with all others, because of space, caging, time limitations and for social reasons. In particular, we did not want to pair monkeys that were not closely bonded. We also refrained from forming mixed-sex pairings because Bolivian squirrel monkey males typically co-habit with females only during the breeding season (Leger, Mason \& Fragaszy, 1981; Mendoza, Lowe \& Levine, 1978); therefore, mixed-sex pairings would both be unnatural and would have come with a risk of aggression and injury.

Table 1

Age, Sex and Relatedness of Squirrel Monkey Pairs

\begin{tabular}{cccccc}
\hline Subject 1 & Age (years) & Subject 2 & Age (years) & Kinship & Sex \\
\hline AL & 6 & OA & 6 & 0.07 & Female \\
SE & 6 & ME & 6 & 0 & Female \\
BG & 5 & MN & 6 & 0.25 & Female \\
FY & 18 & DW & 17 & 0 & Male \\
PE & 6 & RS & 4 & 0.02 & Male \\
\hline
\end{tabular}

Note: Pairs are displayed in rows.

\section{Procedures}

Prior to the study, we determined that our subjects could discriminate among all possible pairings of numbers of food items that they would be exposed to during the game. This is important as there is 
evidence that, even with such small numbers, squirrel monkeys do not always successfully discriminate pairings with large ratio differences (i.e., 2 vs 3 or 3 vs 4; see Gazes, Billas, \& Schmitt, 2018). To test this, subjects were presented with forced choice discriminations of all possible quantities that they would see in the task, excluding any options with no rewards; we assumed that some rewards were preferred over no rewards. Subjects could participate in the three games only if they met our criterion of selecting the greater quantity at least $80 \%$ of the time on two consecutive sessions; thus, all subjects tested met criterion by consistently choosing the greater number of rewards on all possible pairings.

Two token types, differentiated by color or pattern, represented the two outcomes for each game (Figure 1). Although the tokens differed by color (e.g., black/white) and/or pattern (patterned/plain) within a game, across the different games, differently shaped, colored and patterned tokens were used to make it as clear as possible to the subjects that the game had changed. During each game, three of each of the token types were simultaneously presented to each of the paired subjects in identical small bowls (i.e., one bowl containing a total of six tokens for each subject; bowl diameter 4.5 in., depth 2 in.; bowl color also changed with each game). This was done to ameliorate possible side biases if only two tokens were presented.

During testing, the experimenter first shook the bowls containing the tokens, both to randomize them and to attract the subjects' attention, then held them within reach of each subject at the experimenter's shoulder width apart, one in front of each monkey in the pair. To pick an option, subjects had to select a token, pull it through the mesh of their cage, and drop or push it back through the enclosure mesh (to avoid extinguishing the behavior, which was challenging for the monkeys, we also accepted tokens that were not pulled completely through the mesh before being dropped). In studies with other species, subjects returned tokens to the experimenter's hand, but squirrel monkeys were uncomfortable with these interactions, so we used this adapted procedure to make it as easy as possible for the subjects. Previous work indicates that such adapted procedures yield equivalent results in other behavioral and cognitive tasks (Freeman et al., 2013; Talbot et al., 2011). If subjects took two tokens, which happened fairly infrequently, the first token pulled through the mesh and dropped back out was counted as their chosen token.

After one squirrel monkey had taken a token, its partner had up to $5 \mathrm{~s}$ to select a token or the trial was terminated (on the basis that tokens must be returned at approximately the same time to count as a joint action). Once both subjects had a token, they had up to $30 \mathrm{~s}$ to return it before the trial was terminated. When both monkeys in the pair had completed the exchange, the experimenter showed the monkeys the two selected tokens by holding each token in one of her hands and simultaneously raising them toward the front of the subjects' test enclosure for three seconds. Rewards (marshmallow pieces) were then manually delivered to the subjects by the experimenter. To maximize the possibility that subjects saw their partner's outcomes, rewards were distributed sequentially, with the experimenter handing the first reward, withdrawing her hand, then handing the second reward, etc., until all rewards had been given. If both subjects received a reward, both hands were extended simultaneously. If one subject was not to receive a reward, the experimenter kept her hand withdrawn. In this way, subjects got rewards simultaneously, but it was as obvious as we could make it when one was still receiving rewards and the other was not. As related work has shown that primates show a "leader-follower", such that one partner makes a decision and the other follows their lead (Bullinger, Wyman, Melis, \& Tomasello, 2011; see also Brosnan et al., 2011; Smith et al., unpublished data), for each interaction we recorded which monkey made the first move. This allowed us to determine whether order of play had any influence on squirrel monkeys' decisions.

Test sessions consisted of 20 trials, but were terminated if either monkey failed to return a token for 5 min. Sessions were run until each pair completed 300 trials of each game. Subjects completed three different games, the AG, the HDG, and the PDG. The previous work with capuchins presented these games in this sequential order, and for consistency, we replicated this with the squirrel monkeys. The order was chosen because the AG was assumed to be the easiest for the subjects to solve (for the reasons described in the Introduction) and we wished to give them experience with an easier game first to maximize the odds of finding a solution to the more difficult games. All games followed the same procedure, and only the payoffs for each game differed (outlined in Figure 1). An independent researcher coded a randomly selected 20\% of the trial outcomes from video tape, and inter-rater reliability between this researcher and the results GV noted as she collected the data was high (Kappa $=0.89, p<.001)$. 


\section{Statistics}

To assess the outcomes of the economic games, we first used chi-square goodness-of-fit tests to analyze whether the pairs' choice of each outcome differed from what would be predicted by chance (in each game, there were four possible outcomes, so chance was $25 \%$; note that for all three games, all pairs played each of the four potential outcomes within the first 20 trials, so they had early experience with each possible result). If the chi-square analysis was significant, we identified which of the four possible outcomes differed from chance by calculating their standardized residuals ( $s r s$, and those that lay outside $+/-2.58$ were considered statistically significant at $p<.01$ (see Field, 2009). Binomial tests were also performed to isolate whether individuals showed a particular token preference (chance set at 50\%).

Trials in which subjects selected tokens in a sequential manner were analyzed to assess whether the first player's choice influenced the second player's choice using generalized linear mixed effect models (GLMM) run in R (R Development Core Team, 2015) using lme4 (Bates et al., 2015). We constructed 'response order' models that included the second player's choice as the dependent variable and the first player's choice as the predictor variable, with who played first and second entered as random effects. Likelihood ratio tests were performed to compare our response order models against null models with the same structure, except for the exclusion of first choice as a predictor. Models were constructed using the binomial distribution and logit function.

Finally, we reanalyzed capuchin monkeys' performances in the manual versions of the AG and HDG (data originally published in Brosnan et al., 2011, 2018). We did so using the same statistical procedures used for the squirrel monkeys (chi-square goodness-of-fit tests), outlined above. Our approach differed from the chi-square test of association used to analyze capuchin data in the original publications. We opted for the chi-square goodness-of-fit test as we were interested in whether strategy use differed from our expected distribution based on chance expectation (25\%).

For the AG, capuchin pairs had completed 10 sessions of up to 30 trials each. Variable participation meant that not all capuchins completed the full 30 trials in any given session. Our analyses were limited to these 10 sessions, rather than 300 trials, which were not always completed by the capuchins. Therefore, for a direct comparison between species, we also re-analyzed squirrel monkeys' performances during only their first 10 sessions. In the HDG, capuchins completed 300 trials, enabling us to compare the two species on a comparable number of trials, rather than sessions. We used GLMMs to assess whether capuchins and squirrel monkeys differed in the number of times they played the outcome of interest in these games (StagStag play in the AG during a comparable number of test sessions, and Hawk-Dove/Dove-Hawk in the HDG during a comparable number of trials). In our full model, species was entered as the predictor variable, and in our null model, this predictor was omitted. In all models, subject 'pair' was entered as a random effect and likelihood ratio tests were run to assess model fit to the data. As we were using count data, models were constructed using the Poisson distribution (see SM for the squirrel monkey raw data and example R codes).

\section{Results}

\section{Assurance Game}

Across 300 trials, all five of the pairs' task performance significantly differed from chance (Table 2 ). The three female pairs showed a significant preference for Stag-Stag (AL-OA: $s r=3.7, p<.001$; SEME: $s r=8.66, p<.001$; MN-BG: $s r=4.39, p<.001$; see Figure 2). The fourth pair, FY-DW preferentially played Hare-Hare and Hare-Stag. Only the Hare-Hare outcome had a significant standardized residual values $(s r=3.46, p<.001)$; however, this outcome was clearly driven by one male's preference for playing Hare (FY played Hare on 198 trials, binomial: $p<.0001$ ) while his partner played at random (DW played Hare on 167 trials, binomial: $p=.057$ ). The remaining pair's (RS-PE) play of each outcome did not differ from chance (standardized residuals lay within $+/-2.58$ ), although RS preferred the Stag token (played on 174 trials, binomial: $p=.007)$. We saw no significant change in performance when comparing the first and last 100 trial outcomes (Wilcoxon Signed Ranks test: $W=-0.075, p=.940$ ). Finally, our 'response order' 
model did not significantly differ from our null model $\left(\chi^{2}(1, N=10)=0.544, p=.641\right)$, indicating that the first subject's token choices did not have a statistically significant influence on the subsequent choices of their partners (i.e., these data provide no evidence of a leader-follower dynamic).

Table 2

Squirrel Monkey Pairs' Responses According to Game

\begin{tabular}{|c|c|c|c|c|c|c|c|}
\hline \multicolumn{8}{|c|}{ Assurance Game } \\
\hline Pair & Sex & Stag-Stag & Hare-Hare & Hare-Stag & Stag-Hare & $x^{2}$ & $p$-value \\
\hline AL-OA & $\mathrm{F}$ & 107 & 59 & 73 & 61 & 19.73 & .0002 \\
\hline FY-DW & M & 40 & 105 & 95 & 60 & 36.67 & $<.0001$ \\
\hline SE-ME & $\mathrm{F}$ & 150 & 32 & 67 & 51 & 108.19 & $<.0001$ \\
\hline MN-BG & $\mathrm{F}$ & 113 & 46 & 80 & 61 & 33.41 & $<.0001$ \\
\hline RS-PE & M & 90 & 61 & 65 & 84 & 8.03 & .045 \\
\hline \multicolumn{8}{|c|}{ Hawk-Dove Game } \\
\hline Pair & & Dove-Dove & Hawk-Hawk & Dove-Hawk & Hawk-Dove & $x^{2}$ & $p$-value \\
\hline AL-OA & $\mathrm{F}$ & 89 & 57 & 86 & 68 & 9.2 & .0267 \\
\hline FY-DW & M & 82 & 55 & 95 & 68 & 11.97 & .0075 \\
\hline SE-ME & $\mathrm{F}$ & 65 & 69 & 89 & 77 & 4.48 & .2141 \\
\hline MN-BG & $\mathrm{F}$ & 100 & 57 & 45 & 98 & 31.71 & $<.0001$ \\
\hline RS-PE & M & 69 & 71 & 73 & 87 & 2.67 & .4453 \\
\hline \multicolumn{8}{|c|}{ Prisoner's Dilemma Game } \\
\hline Pair & & $\begin{array}{l}\text { Cooperate- } \\
\text { Cooperate }\end{array}$ & Defect-Defect & $\begin{array}{c}\text { Defect- } \\
\text { Cooperate }\end{array}$ & $\begin{array}{c}\text { Cooperate- } \\
\text { Defect }\end{array}$ & $x^{2}$ & $p$-value \\
\hline AL-OA & $\mathrm{F}$ & 89 & 56 & 88 & 67 & 10.53 & .0146 \\
\hline FY-DW & M & 57 & 76 & 50 & 117 & 36.19 & $<.0001$ \\
\hline SE-ME & $\mathrm{F}$ & 84 & 57 & 89 & 70 & 8.35 & .0393 \\
\hline MN-BG & $\mathrm{F}$ & 79 & 85 & 57 & 79 & 6.08 & .1078 \\
\hline RS-PE & M & NA & NA & NA & NA & NA & NA \\
\hline
\end{tabular}

Note: Scores indicate the number of trials in which each pair selected each possible outcome (all 300 trials reported). The first player listed under "Pair" played the first listed outcome (i.e., AL played Hare in Hare-Stag and Stag in Stag-Hare). Significant chi-square results $(p<.05)$ indicated in bold. ' $\mathrm{F}$ ' denotes female pairs and 'M' male.

\section{Hawk-Dove Game}

In the anti-coordination game, three of the pairs' task performance significantly differed from chance (Table 2). MN-BG primarily played Dove-Dove and Hawk-Dove (Dove-Dove: $s r=2.89, p<.01$; Hawk-Dove: $s r=2.66, p<.01$ ), with BG significantly preferring Dove (on 196 trials, binomial $=p<.001$ ) and her partner MN playing at random. Although two other subjects preferred the Dove token (FY and AL), in no other pair was a single game outcome significant at $\alpha<.01$. We saw no significant change in performance when comparing the first and last 100 trial outcomes (Wilcoxon Signed Ranks test: $W=$ $0.131, p=.896)$. Again, our 'response order' model did not significantly differ from our null model $\left(\chi^{2}(1\right.$, $N=10)=4.801, p=.091)$. 
Vale et al. 40
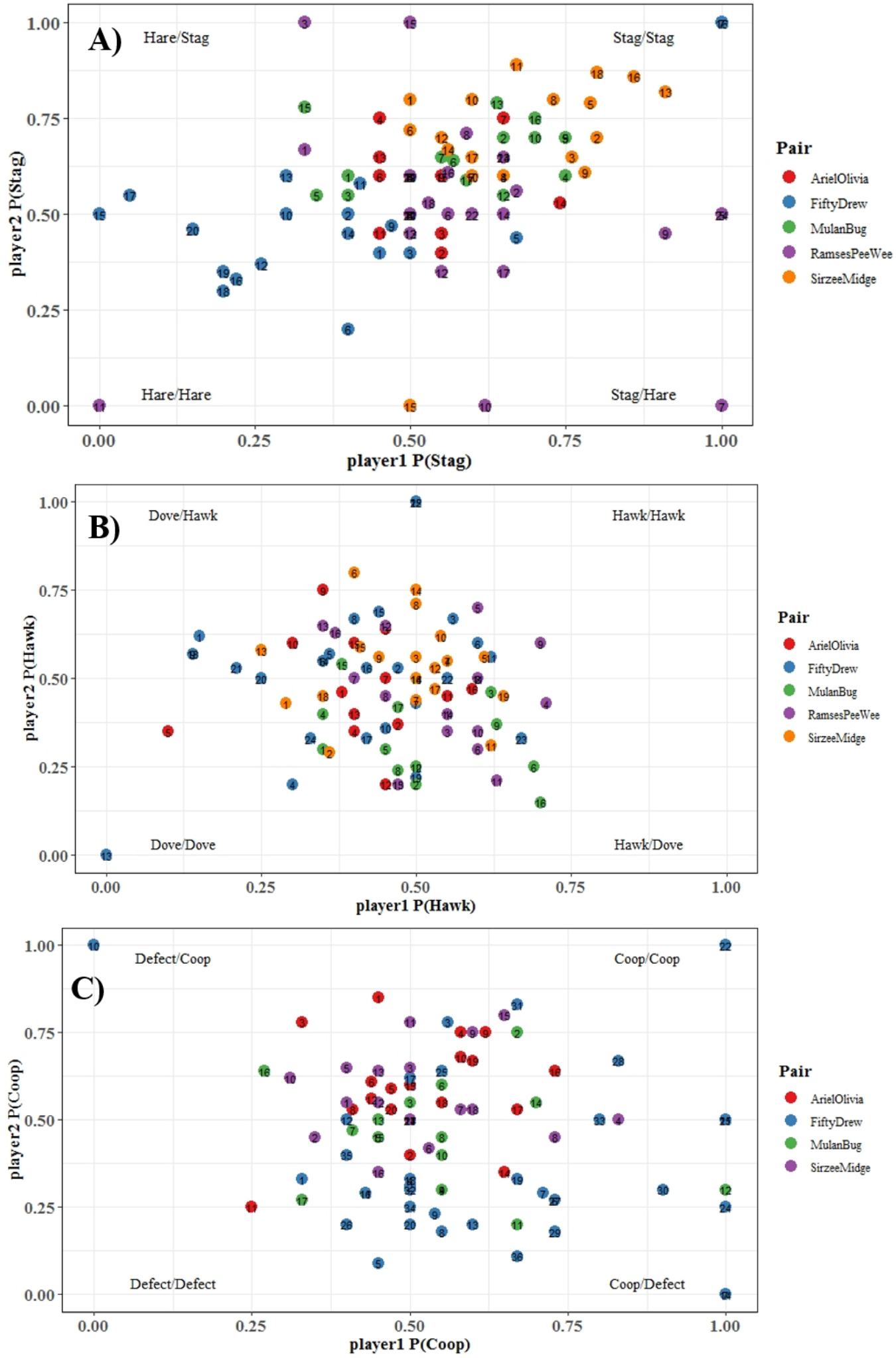

Pair

- ArielOlivia

- FiftyDrew

- MulanBug

- SirzeeMidge

Figure 2. Squirrel monkeys' responses in the Assurance Game (A), Hawk-Dove Game (B), and the Prisoner's Dilemma (C). Numbers within the circles represent the session number. $\mathrm{X}$ and $\mathrm{Y}$-axis reflect the proportion of the response within a session by Player 1 and Player 2, respectively. Pairs that completed less than 20 trials per session completed more than 15 sessions per game. 


\section{Prisoner's Dilemma Game}

In this cooperative game, FY-DW showed a significant preference for the Cooperate-Defect outcome (Table 2; $s r=4.85, p<.001$ ), with FY preferring the Cooperate option (174 plays in 300 trials, binomial: $p=.007$ ) and DW preferring the Defect option (DW, 193 plays in 300 trials, binomial: $p<.001$ ). Two females (OA and ME), from different pairs, showed individual preferences for the Cooperate option (binomial: $p$ s <.05), but in no case did other pairs' game outcomes differ from chance $($ at $\alpha<.01)$. We again saw no significant change in performance when comparing the first and last 100 trial outcomes (Wilcoxon Signed Ranks test: $W=-0.259, p=.795$ ). Our 'response order' model did not significantly differ from our null model $\left(\chi^{2}(1, N=8)=0.174, p=.677\right)$.

\section{Comparison with Capuchin Performance (Assurance and Hawk-Dove Game)}

Six capuchin monkey pairs previously participated in the manual version of the AG (see Brosnan et al., 2011 for full details of the methodology). Two capuchin pairs' performances statistically differed from chance (Wren-Lilly: $\chi^{2}(3, N=2)=10, p=.0186$; Griffin-Drella: $\left.\chi^{2}(3, N=2)=14.19, p=.0027\right)$, with both pairs primarily playing Stag-Stag ( $s r=2.73$ and $s r=3.13$, respectively, $p s<.01$ ). No other pairs' game outcomes significantly differed from chance (at $\alpha<.01$ ). Considering just the first 10 sessions for our squirrel monkeys (equivalent to capuchins), we again found that the three female pairs (of five total pairs) showed significant preferences for playing Stag-Stag (AL-OL: $\chi^{2}(3, N=2)=19.40, p=.0002$; ML-BG: $\chi^{2}(3, N=2)=17.09, p=.0007$; SE-ME: $\chi^{2}(3, N=2)=56.99, p<.0001$, srs $\left.>2.58\right)$. Our full model, including species as a predictor, did not significantly differ from our null model $\left(\chi^{2}(1, N=22)=0.660, p\right.$ $=.4167)$, indicating that, although a greater percentage of squirrel monkey pairs found the Stag-Stag outcome, in our sample, species was not a significant predictor of Stag-Stag play.

Four capuchin pairs previously participated in the manual version of the HDG (Brosnan et al., $2018)$. When playing this game, only one pair's performance significantly differed from chance $\left(\chi^{2}(3, N=\right.$ $2=9.31, p=.0254)$, but even in this case, none of the game outcomes were significant at $\alpha<0.01$. Not surprisingly, considering that none of the squirrel monkeys settled on a single game outcome either, when assessing species differences in the play of the NEs (one player plays Dove and the other Hawk), we again found that our full model including species as a predictor did not significantly differ from our null model that omitted this variable $\left(\chi^{2}(1, N=18)=0.0712, p=.790\right)$.

\section{Discussion}

Previous work using these three games derived from experimental economics has been useful in uncovering the similarities and differences in primate species' decision-making outcomes. In the current study, we added squirrel monkeys, a neotropical primate that is both closely phylogenetically related to, and often found sympatrically with, capuchin monkeys, and shares many morphological, behavioral, and cognitive traits with capuchins. However, there is far less evidence of cooperation in squirrel monkeys as compared to capuchins, making them an ideal comparison species to study the social context of decision making. This comparison provides some initial evidence to help determine whether squirrel monkeys cooperate when given the proper environmental contingencies. Indeed, providing some support for this, three of the five pairs coordinated in the Assurance Game (AG). Although our small sample size reduces our power to detect species differences, both our analysis and a qualitative assessment indicate that they perform comparably to capuchins in the manual task. In subsequent games, however, very few of the squirrel monkeys established any consistent pattern of play that was statistically different from chance. We consider each of these findings in more detail below.

We were surprised by the squirrel monkeys' ability to find the coordinated NE in the AG. We did not expect this, because there is comparatively very little evidence of cooperation or coordination in squirrel monkeys, especially relative to capuchins (see Introduction). We see several non-mutually exclusive possible explanations for this result. First, and most obviously, it may be that squirrel monkeys do 
coordinate, but that we have simply not found the evidence yet. Our (admittedly subjective) assessment is that there is far less research effort on squirrel monkeys than capuchins, particularly in field conditions. This means that it is very likely that there are many interesting behaviors that we have simply not yet documented. For example, it required years of observation before some apparently widespread behaviors (i.e., group patrols) were seen in chimpanzee field sites. Indeed, squirrel monkeys do reconcile and share food, all behaviors that are highly social and are argued to be related to cooperation and coordination (Pereira et al., 2000; Stevens, 2004). On the other hand, squirrel monkeys do not respond negatively to inequity (Talbot et al., 2011), also a behavior typically associated with cooperation.

Second, our findings could suggest that the best paying solution to the AG can easily be learned, even by species that do not typically cooperate or coordinate. One simple way for animals to find the coordinated, payoff-dominant NE in this game is to develop a preference for the highest paying Stag token, without understanding that their payoffs depended on the actions of their partners as well as on their own. In principle, this could help to explain why the two species performed similarly. If the capuchin monkeys do tend to cooperate more frequently, and understood that outcomes were jointly determined, but struggled to determine their partners' motives, we would expect a lower frequency of finding the cooperative outcomes. On the other hand, if the squirrel monkeys understood the game as a reward maximization task, it may have been easier to solve because they would not have had to take their partner's play into account. Even if feasible, we hesitate to endorse this view as we do not know that the capuchins understood the AG as a coordination task rather than a reward maximization task.

There is, however, reason to speculate that the squirrel monkeys learned the payoff-dominant, coordinated outcome by preferring to exchange the higher paying Stag token. Notably, in no situation did we find evidence of the squirrel monkeys changing their pattern of play based on what the first player chose, even though doing so would have been beneficial. This appears to be an important difference from capuchins, for whom second movers adjusted their decision based on the first movers' play in a computerized version of the HDG and PDG (Smith et al., unpublished data). Squirrel monkeys use social information in other contexts (Claidiere, Messer, Hoppitt, \& Whiten, 2013; Hopper et al., 2013), so this implies that either they did not understand the task as a social one, or that they did not use this information to benefit them. Finding that squirrel monkeys did not flexibly change their behavior to match their partner, even though they are sensitive to their partners in other contexts, supports the hypothesis that pairs learned the coordinated outcome by playing the token that could provide them with the highest payoff.

No matter how the squirrel monkeys were finding the coordinated outcome, it is nevertheless notable that they appear to be as likely to solve the task as the capuchins. Aside from the aforementioned differences in rates of cooperation that have been seen in both wild populations and experimental studies, the capuchins studied previously (Brosnan et al., 2011, 2018) are far more experienced in cognitive testing than are these squirrel monkeys, with most having participated in cognitive and behavioral testing daily or nearly daily for many years (well over a decade for most of these subjects). Some of the squirrel monkeys, in contrast, had never participated in a behavioral or cognitive study, and those that had testing experience had participated in only a single study. In our previous work, we found that chimpanzees with more experience were more likely to find the NE than those with less (Brosnan et al., 2011), which would suggest the hypothesis that the capuchins should have outperformed the squirrel monkeys irrespective of natural cooperative tendency. However, this is not what we found, raising some question over how much of an effect prior testing history may have on participants' abilities to solve the AG, and whether it varies by species.

Another curious outcome that we hesitate to discuss in detail, at the risk of drawing more attention than is warranted given the sample size, is that the females coordinated in the AG, but the males did not. Again, it is highly likely that this is coincidence. If indeed a cognitive testing experience effect exists, this is especially so as the females had previously participated in a cognitive study, and the males had not (see Methods). Nonetheless, female Bolivian squirrel monkeys are dominant and philopatric, form kin-based affiliative bonds and coalitions, engage in allomothering, and even compete with other matrilines over resources (Boinski \& Croop, 1999; Boinski et al., 2002; Williams et al., 1994). This pattern of female bonding does not generalize to all squirrel monkeys; $S$. oerstedi do not appear to form female affiliative 
coalitions or female dominance hierarchies, and for them, males rather than females are philopatric (Boinski et al., 2002). Given these differences in female sociality, we would be very interested to see future work comparing $S$. oerstedi and $S$. boliviensis females' cooperation. We cautiously predict that $S$. boliviensis would outperform $S$. oerstedi, which, if supported, would provide evidence for the hypothesis that cooperation has been selected in at least some squirrel monkey species in specific social contexts.

Almost as surprising as the squirrel monkeys' success in the AG was their subsequent inability to find consistent patterns of play in any of the other tasks. Indeed, not only did pairs fail to find the NE, but in the HDG, three individuals showed preferences for playing Dove, which their partners failed to exploit by playing Hawk (which would have given them the highest paying outcome). Even though one pair in the PDG did appear to exploit their partner's preference for Cooperate by playing Defect, there was no evidence that second players altered their decision after seeing what the first player chose. Therefore, despite this latter pair's statistically significant preference, we have no evidence of flexible play that was contingent on the play of partners. We see several possible reasons for their lack of a consistent response with the HDG and PDG.

First, it appears that the NE are harder to find in the HDG and PDG. Although these games were chosen because we can use the same procedure and the same range of outcomes across the decision situations (i.e., the lowest payoff is 0 or 1 and the highest is 4), the NE and best paying strategy differ across the games. We hypothesize that the HDG and PDG are harder because there is not a single, mutually beneficial highest paying outcome, such as the payoff-dominant NE in the AG, and because the outcomes are unequal between partners (although note that we have no evidence that squirrel monkeys notice or care about reward inequity with group mates; Freeman et al., 2013; Talbot et al., 2011). In addition, some require mismatched responses, which may be difficult for animals to learn (Martin, Bhui, Bossaerts, Matsuzawa, \& Camerer, 2014). Moreover, although all of our subjects passed a pre-test to discriminate each possible reward pairing prior to the study, the payoff differences in the AG (1 vs 4) were larger than in the other two games (e.g., 3 vs 4 and 2 vs 4), which may have made it more challenging for the monkeys to find (or care about) the payoff dominant outcome in the other games. Finally, in the AG only, one of the token options changed in payoff value (the Hare reward was constant). In contrast, for the HDG and PDG, both tokens changed in payoff value depending upon partner's choices, which may have added to the complexity of these games (e.g., this may have placed greater demands on memory as our monkeys had to track not one, but two changeable payoffs).

It is also possible that inhibiting a preference for the highest paying token, which was required for cooperation to emerge in the PDG, as well as for settling on one or both asymmetric NE in the HDG, may have hindered participants' performances in these games. We nevertheless note two reasons why this may not have played a large role. First, these monkeys are capable of learning to inhibit the larger of two quantities in reverse-reward contingency tasks (Anderson, Awazu, \& Fujita, 2000). Second, most participants showed individual preferences for the highest paying token only when it was mutually beneficial (in the AG). During the HDG and PDG, among subjects who showed a preference for one token over the other, the majorities' preference was for the lower paying token option (Dove and Cooperate). This suggests that at least some of our sample inhibited selection of the token that could have yielded the most rewards (at least in some contexts; in others, the token was associated with the zero outcome, which would have made it particularly aversive).

These added difficulties may have meant that, regardless of whether they could have solved the task, they could have also been less motivated to do so. Moreover, nearly all choices resulted in at least some reward, even if not the maximum. Our monkeys were never food deprived, so these rewards were on top of their daily diet, which may have lowered their motivation to work hard enough to gain a few extra rewards when some were practically guaranteed. Indeed, our monkeys often failed to finish all 20 trials in a session, indicating that the rewards were not motivating enough to overcome their often distractible natures (see also Fragaszy, 1981, 1985). Of course, this was also the case in the AG, in which the majority of pairs coordinated, but it may be that, in the easier context, they were able to settle on a consistent pattern of play despite low motivation. 
Indeed, squirrel monkeys are not the only species that have found the HDG and PDG more challenging than the AG. Rhesus monkeys, for example, found the payoff dominant NE in the AG both when they could and could not see their partner's choices, yet in the HDG they only settled on a consistent pattern of play, also the NE, when they could see what their partner had played (Brosnan et al., 2018). Capuchins similarly have had some trouble finding any consistent pattern of play the HDG and PDG. In the HDG, they found the NE in a computerized task, but failed to do so in a manual version, even though they found the NE in the AG in both manual and computerized versions (Brosnan et al., 2011; 2018). In the PDG, capuchins also took more trials to develop stable patterns of play, fewer pairs did so than in other games, and the outcomes they found differed across pairs, unlike in other games (Smith et al., unpublished data). This suggests that both Old and New World monkeys have greater difficulty in finding outcomes that require anti-coordination or costly cooperation than those that require payoff dominant coordinated or matched responses, at least in the context of the economic games that we presented them. However, as we have discussed, how easily the NE is found in each game may reflect slight changes in the demands of each game (e.g., in motivation, memory and inhibition) rather than species' propensities in these regards.

It is important to note that we were studying the question of whether untrained subjects could find the NE, or learn to find it, not whether they could play them once they were trained on the payoff contingencies. These are both important questions that should be addressed, but, for this work, we chose to explore the question of whether pairs developed consistent patterns of play when they were initially naïve to the games' outcomes for two reasons. First, we wished to establish how subjects responded when their behavioral outcomes were contingent on their partner's responses in a novel situation. Humans face similar situations, and it is important to know how (or whether) subjects make choices in these situations. Second, there is a risk that, despite our best efforts, teaching them the contingencies will accidentally train them to play a specific outcome. For example, capuchins solve the AG by developing a preference for the highestpaying outcome (Brosnan et al., 2012). In that particular case, the subjects learned the contingencies on their own, but had we provided pre-training on the token values, they would almost certainly have settled on the same strategy, making it impossible to determine whether they learned the contingencies during the game or simply played the token that was most valuable during training. It will be interesting to see in future work how responses may differ if subjects are trained on the game contingencies prior to their participation. In particular, we are curious whether they will be more successful in finding the NE in the more complex games (HDG and PDG) if they receive training prior to testing.

To our surprise, at least some S. boliviensis were able to coordinate in the AG and did so at similar rates as capuchins in an exchange task. However, this ability to find the NE did not extend to the other games. This research opens a number of future avenues. First, coordination games can be solved by species that do not typically cooperate. Work is needed to determine whether they are adopting the same highest paying actions through associative learning or cooperating in ways that have yet to be recognized (although we note that these are not mutually exclusive). We encourage further work in this area to identify any previously unrecognized situations of coordination in the wild and conditions that may encourage the acquisition or expression of coordination. In addition, we hope that others with the ability to test squirrel monkeys in a computerized set-up will replicate these tasks to see if, as with capuchins, they do better in computerized versions of the task. This will improve our comparison and potentially identify other situations in which the monkeys can find the NE. These results emphasize the utility of a comparative approach in understanding the roots of behavior to identify unexpected similarities between species as well as potential differences in how they achieve these outcomes.

\section{Acknowledgements}

We are grateful to Bethany Brock and to all the other care staff at the KCCMR. We are also extremely grateful to Mackenzie Smith, who developed the R code used in our analyses and kindly shared it with us. This work was supported by an NSF grant (SES 1123897 awarded to Sarah Brosnan, Steve Schapiro, Larry Bart Wilson and Mike Beran) and an NIH grant (P40-OD010938-38 awarded to Dr. Chris Abee). 


\section{References}

Alfaro, J. W. L., Silva, J. D., \& Rylands, A. B. (2012). How different are robust and gracile capuchin monkeys? An argument for the use of Sapajus and Cebus. American Journal of Primatology, 74, 273-286.

Bates, D., Mächler, M., Bolker, B., \& Walker, S. (2015). Fitting linear mixed-effects models using lme4. Journal of Statistical Software, 67, 1-48.

Boinski, S. (1999). The social organizations of squirrel monkeys: Implications for ecological models of social evolution. Evolutionary Anthropology: Issues, News, and Reviews, 8, 101-112.

Boinski, S., \& Cropp, S. J. (1999). Disparate data sets resolve squirrel monkey (Saimiri) taxonomy: Implications for behavioral ecology and biomedical usage. International Journal of Primatology, 20, 237-256.

Boinski, S., Sughrue, K., Selvaggi, L., Quatrone, R., Henry, M., \& Cropp, S. (2002). An expanded test of the ecological model of primate social evolution: Competitive regimes and female bonding in three species of squirrel monkeys (Saimiri oerstedii, S. boliviensis and S. sciureus). Behaviour, 139, 227-261.

Brosnan, S. F. (2011). What do capuchin monkeys tell us about cooperation? In D. R. Forsyth \& C. L. Hoyt (Eds.), For the greater good of all: Perspectives on individualism, society, \& leadership (p. 11-28). New York: Palgrave Macmillan Publishers.

Brosnan, S. F., \& de Waal, F. B. (2014). Evolution of responses to (un)fairness. Science, 346, 1251776.

Brosnan, S. F., Parrish, A., Beran, M. J., Flemming, T., Heimbauer, L., ...Wilson, B. J. (2011). Responses to the Assurance Game in monkeys, apes, and humans using equivalent procedures. Proceedings of the National Academy of Sciences, 108, 3442-3447.

Brosnan, S. F, Price, S. A., Leverett, K., Prétôt, L., Beran, M. J., \& Wilson, B. J. (2018). Human and monkey responses in a symmetric game of conflict with asymmetric equilibria. Journal of Economic Behavior \& Organization, 142, 293-306.

Brosnan, S. F., Wilson, B. J., \& Beran, M. J. (2012). Old World monkeys are more similar to humans than New World monkeys when playing a coordination game. Proceedings of the Royal Society of London B: Biological Sciences, 279, 1522-1530.

Bullinger, A. F., Wyman, E., Melis, A. P., \& Tomasello, M. (2011). Coordination of chimpanzees (Pan troglodytes) in a stag hunt game. International Journal of Primatology, 32, 1296-1310.

Chalmeau, R., Lardeux, K., Brandibas, P., \& Gallo, A. (1997). Cooperative problem solving by orangutans (Pongo pygmaeus). International Journal of Primatology, 18, 23-32.

Claidiere, N., Messer, E. J., Hoppitt, W., \& Whiten, A. (2013). Diffusion dynamics of socially learned foraging techniques in squirrel monkeys. Current Biology, 23, 1251-1255.

Clements, K. C., \& Stephens, D. W. (1995). Testing models of non-kin cooperation: Mutualism and the prisoner's dilemma. Animal Behaviour, 50, 527-535.

Crawford, M. P. (1937). The cooperative solving of problems by young chimpanzees. Baltimore: Johns Hopkins Press.

Crawford, M. P. (1941). The cooperative solving by chimpanzees of problems requiring serial responses to color cues. The Journal of Social Psychology, 13, 259-280.

Cronin, K. A., Kurian, A. V., \& Snowdon, C. T. (2005). Cooperative problem solving in a cooperatively breeding primate (Saguinus oedipus). Animal Behaviour, 69, 133-142.

de Waal, F. B. (2000). Attitudinal reciprocity in food sharing among brown capuchin monkeys. Animal Behaviour, 60, 253-261.

de Waal, F. B., \& Berger, M. L. (2000). Payment for labour in monkeys. Nature, 404, 563.

de Waal, F. B., \& Davis, J. M. (2003). Capuchin cognitive ecology: Cooperation based on projected returns. Neuropsychologia, 41, 221-228.

Deaner, R. O., Isler, K., Burkart, J., \& van Schaik, C. (2007). Overall brain size, and not encephalization quotient, best predicts cognitive ability across non-human primates. Brain, Behavior and Evolution, 70, 115-124.

di Bitetti, M. S. (1997). Evidence for an important social role of allogrooming in a platyrrhine primate. Animal Behaviour, 54, 199-211

Field, A. (2009). Discovering statistics using SPSS. London: Sage Publications.

Fleagle, J. G. (1999). Primate adaptation \& evolution. New York: Academic Press.

Fragaszy, D. M. (1981). Comparative performance in discrimination learning tasks in two New World Primates (Saimiri sciureus and Callecebus moloch). Animal Learning and Behaviour, 9, 127-134.

Fragaszy, D. M. (1985). Cognition in squirrel monkeys: A contemporary perspective. In L. A. Rosenblum \& C. L. Coe (Eds.), Handbook of squirrel monkey research (pp. 55-98). Boston, M: Springer.

Fragaszy, D. M., Visalberghi, E., \& Fedigan, L. M. (2004). The complete capuchin: The biology of the genus Cebus. Cambridge U.K.: Cambridge University Press. 
Freeman, H. D., Sullivan, J., Hopper, L. M., Talbot, C. F., Holmes, A. N.,...Brosnan, S. F. (2013). Different responses to reward comparisons by three primate species. PloS One, 8, e76297.

Gardner, R., Corbin, T., Beltramo, J., \& Nickell, G. (1984). The prisoner's dilemma game and cooperation in the rat. Psychological Reports, 55, 687-696.

Gazes, R. P., Billas, A. R., \& Schmitt, V. (2018). Impact of stimulus format and reward value on quantity discrimination in capuchin and squirrel monkeys. Learning \& Behavior, 46, 89-100.

Green, L., Price, P. C., Hamburger, M. E. (1995). Prisoner's dilemma and the pigeon: Control by immediate consequences. Journal of the Experimental Analysis of Behavior, 64, 1-17.

Greenberg, J. R., Hamann, K., Warneken, F., \& Tomasello, M. (2010). Chimpanzee helping in collaborative and noncollaborative contexts. Animal Behaviour, 80, 873-880.

Hare, B., Melis, A. P., Woods, V., Hastings, S., \& Wrangham, R. (2007). Tolerance allows bonobos to outperform chimpanzees on a cooperative task. Current Biology, 17, 619-623.

Haroush, K., \& Williams, Z. M. (2015). Neuronal prediction of opponent's behavior during cooperative social interchange in primates. Cell, 160, 1233-1245.

Hartwig, W., Rosenberger, A. L., Norconk, M. A., \& Owl, M. Y. (2011). Relative brain size, gut size, and evolution in New World monkeys. The Anatomical Record, 294, 2207-2221.

Hopper, L. M., Holmes, A. N., Williams, L. E., \& Brosnan, S. F. (2013). Dissecting the mechanisms of squirrel monkey (Saimiri boliviensis) social learning. PeerJ, 1, e13.

Leger, D. W., Mason, W. A., \& Fragaszy, D. M. (1981). Sexual segregation, cliques, and social power in squirrel monkey (Saimiri) groups. Behaviour, 76, 163-181.

Li, G., \& Wood, R. I. (2017). Male rats play a repeated donation game. Physiology \& Behavior, 174, 95-103.

Manson, J. H., Navarrete, C. D., Silk, J. B., \& Perry, S. (2004). Time-matched grooming in female primates? New analyses from two species. Animal Behaviour, 67, 493-500.

Marroig, G. (2007). When size makes a difference: Allometry, life-history and morphological evolution of capuchins (Cebus) and squirrels (Saimiri) monkeys (Cebinae, Platyrrhini). BMC Evolutionary Biology, 7, 20.

Martin, C. F., Bhui, R., Bossaerts, P., Matsuzawa, T., \& Camerer, C. (2014). Chimpanzee choice rates in competitive games match equilibrium game theory predictions. Scientific Reports, 4, 5182.

Melis, A. P., Hare, B. \& Tomasello, M. (2006a). Chimpanzees recruit the best collaborators. Science, 311, 1297-1300.

Melis, A. P., Hare, B. \& Tomasello, M. (2006b). Engineering cooperation in chimpanzees: Tolerance constraints on cooperation. Animal Behaviour, 72, 275-286.

Mendoza, S. P., Lowe, E. L., \& Levine, S. (1978). Social organization and social behavior in two subspecies of squirrel monkeys (Saimiri sciureus). Folia Primatologica, 30, 126-144.

Parrish, A. E., Brosnan, S. F., Wilson, B. J., \& Beran, M. J. (2014). Differential responding by rhesus monkeys (Macaca mulatta) and humans (Homo sapiens) to variable outcomes in the assurance game. Animal Behavior and Cognition, 1, 215-229.

Pereira, M. E., Schill, J. L., \& Charles, E. P. (2000). Reconciliation in captive Guyanese squirrel monkeys (Saimiri sciureus). American Journal of Primatology, 50, 159-167.

Perry, S., Manson, J. H., Dower, G., \& Wikbert, E. (2003). White-faced capuchins cooperate to rescue a groupmate from a Boa constrictor. Folia Primatologica, 74, 109-111.

R Development Core Team. (2015) R: A language and environment or statistical computing R Foundation for Statistical Computing, Vienna, Austria.

Reader, S. M., \& Laland, K. N. (2002). Social intelligence, innovation, and enhanced brain size in primates. Proceedings of the National Academy of Sciences, 99, 4436-4441.

Rose, L. M. (1997). Vertebrate predation and food-sharing in Cebus and Pan. International Journal of Primatology, 18, 727-765.

Sabbatini, G., Vizioli, A. D. B., Visalberghi, E., \& Schino, G. (2012). Food transfers in capuchin monkeys: An experiment on partner choice. Biology Letters, 8, 757-759.

Scarry, C. J. (2017). Male resource defence during intergroup aggression among tufted capuchins. Animal Behaviour, $123,169-178$.

Skyrms, B. (2003). The stag hunt and the evolution of social structure. Cambridge, U.K.: Cambridge University Press.

Smith, M. F., Leverett, K., Wilson, B.J., \& Brosnan, S. F. (unpublished data). Capuchin monkeys (Cebus [sapajus] apella) play Nash equilibria, but their decisions are not influenced by oxytocin.

Smith, M. F., Watzek, J. \& Brosnan, S. F. (2018). The importance of a truly comparative methodology for comparative psychology. International Journal of Comparative Psychology, 31.

Stevens, J. R. (2004). The selfish nature of generosity: harassment and food sharing in primates. Proceedings of the Royal Society of London B: Biological Sciences, 271, 451-456. 
Stevens, J. R., \& Stephens, D. W. (2004). The economic basis of cooperation: Tradeoffs between selfishness and generosity. Behavioral Ecology, 15, 255-261.

Talbot, C. F., Freeman, H. D., Williams, L. E., \& Brosnan, S. F. (2011). Squirrel monkeys' response to inequitable outcomes indicates a behavioural convergence within the primates. Biology Letters, 7, 680-682.

Verbeek, P., \& de Waal, F. B. (1997). Postconflict behavior of captive brown capuchins in the presence and absence of attractive food. International Journal of Primatology, 18, 703-725.

Williams, L., Gibson, S., McDaniel, M., Bazzel, J., Barnes, S., \& Abee, C. (1994). Allomaternal interactions in the Bolivian squirrel monkey (Saimiri boliviensis boliviensis). American Journal of Primatology, 34, 145-156.

Wolfe, J. B. (1934). The effect of delayed reward upon learning in the white rat. Journal of Comparative Psychology, 17, 1-21. 